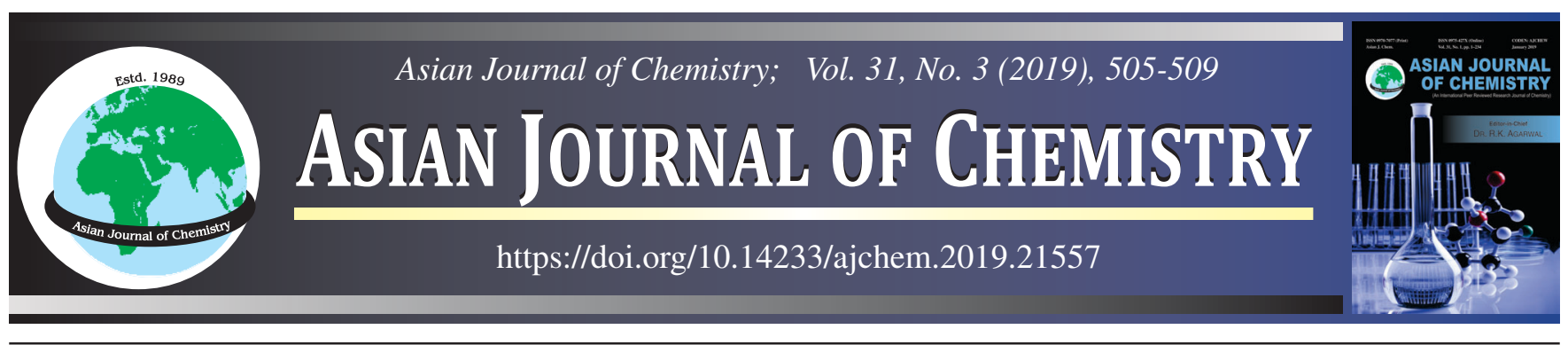

\title{
Structural Dependence of Non-Linear Optical Properties of Molecules Containing Naphthalene Linked to Nitrophenyl Group-A DFT Study
}

AnJu Linda VARGHeSE ${ }^{1, *}$, IgnATIOUS Abraham ${ }^{2}$ and M. GeORGE ${ }^{2}$

${ }^{1}$ Department of Chemistry, Catholicate College, Pathanamthitta-689645, India

${ }^{2}$ Department of Chemistry, Sacred Heart College (Autonomous ), Thevara, Kochi-682013, India

*Corresponding author: E-mail: lindaanju@ gmail.com

Received: 3 July 2018;

Accepted: 22 August 2018;

Published online: 31 January 2019;

AJC-19235

Nonlinear optical (NLO) properties of N-[3-(naphthalene-1-yloxy)butyl]-4-nitroaniline and N-[3-(naphthalene-1-yloxy)butyl]-2,4dinitroaniline have been calculated theoretically. Theoretical calculations were performed with four different hybrid density functional theories (DFT) i.e. BPV86, B3LYP, LSDA and M-06 with 6-31++G(d,p) basis set. The results showed that these molecular systems have large first static hyperpolarizabilities. Moreover, NLO response of these molecular systems decreased considerably when nitrophenyl is replaced by dinitrophenyl group.

Keywords: Nonlinear optical, Hyperpolarizability, Mono-/Dinitroaniline, DFT.

\section{INTRODUCTION}

Nonlinear optical (NLO) materials play a vital role in nonlinear optics and have a great impact on information technology and industrial applications. Theoretical calculations play a significant role in understanding the polarization mechanism of NLO materials and their relation to structural characteristics. Last decade witnessed the development of new nonlinear optical materials of inorganic, organic and semi-organic types. Organic nonlinear optical materials have potentially high nonlinearities and rapid response to light [1-4]. They offer high degree of synthetic flexibility to tune their optical properties through structural modification [5]. Organic frameworks with large delocalized $\pi$-systems have proven to be useful. This is attributed to the fact that $\pi$-electrons are more easily affected by an external optical field as they are relatively loosely bound to the nucleus and that the delocalized orbitals may be extended over the entire molecule giving large and fast polarization [6-9]. The delocalization of $\pi$-electrons can be further enhanced by the addition of donor and accepter groups at the opposite ends of the conjugated system. The strong charge transfer between such groups operating across the entire system considerably adds to the optical nonlinearity of structure [10-12]. The large value of first hyperpolarizability $(\beta)$, which is the measure of nonlinear optical activity, is attributed to intramolecular charge transfer which occurs due to the movement of electron cloud from electron donor to acceptor groups through a $\pi$ conjugated framework [13-16]. The designing of new nonlinear optical materials relies heavily on the theoretical prediction of accurate electro-optical properties for aforementioned systems.

The polarizability of organic frameworks containing naphthalene has been extensively studied with different theoretical methods and found to have good nonlinear response. An efficient method to modulate electron density distribution in this conjugated system is the incorporation of functional groups (spacers) into its backbone. Such studies are done on conjugated oligomers and polymers $[17,18]$. But donor-acceptor systems containing naphthalene incorporated with spacer groups in the backbone are largely unexplored.

The principal aim of this work is to undertake an exhaustive theoretical investigation on the structural, electronic and optical properties of naphthalene framework linked to nitrophenyl group through a spacer groups. The molecules with large hyperpolarizability values can be developed into NLO materials which are having potential applications in the optoelectronic devices of telecommunications, information storage, optical switching and photovoltaic devices like solar cells. We propose to investigate molecules in which naphthalene group is connected to mono-/

This is an open access journal, and articles are distributed under the terms of the Creative Commons Attribution-NonCommercial-ShareAlike 4.0 (CC BY-NC-SA 4.0) International License which allows readers to freely read, download, copy, distribute, print, search, or link to the full texts of its articles and to use them for any other lawful non-commercial purpose as long as the original source is duly acknowledged. 

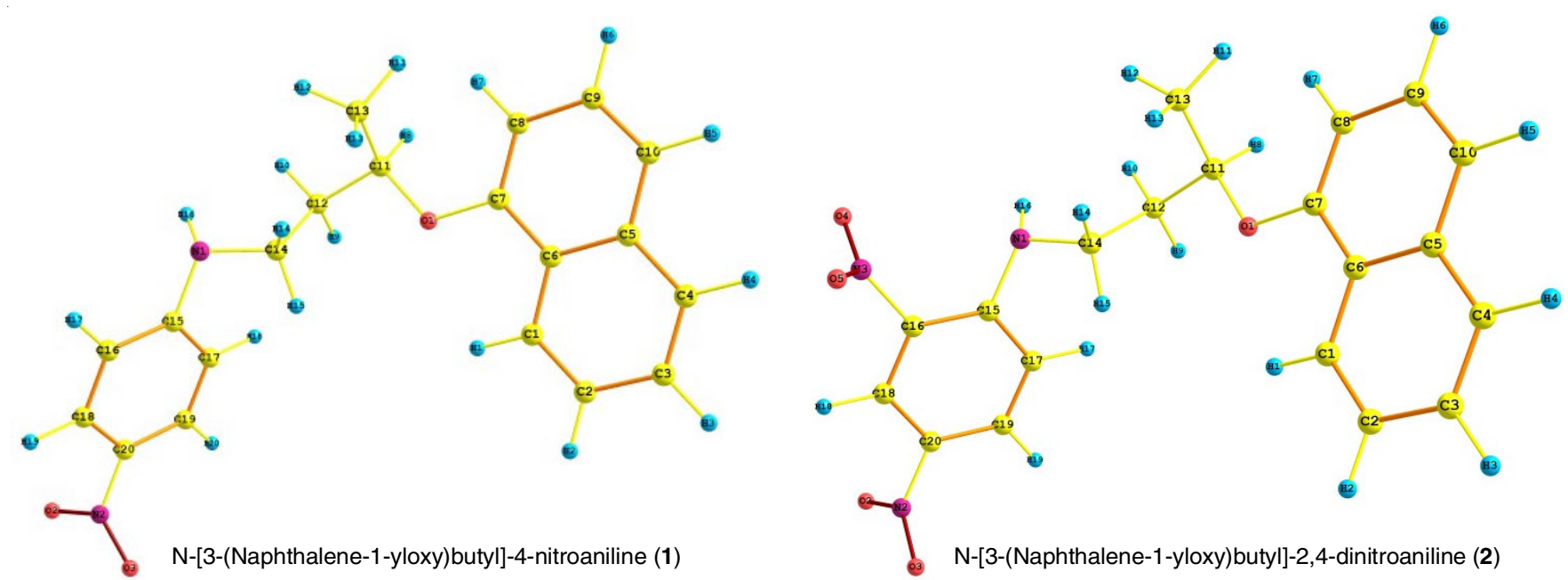

Fig. 1. An atom numbering scheme of compounds $\mathbf{1}$ and $\mathbf{2}$. The carbon, nitrogen, oxygen and hydrogen atoms are indicated by yellow, pink, red and blue colours, respectively

di-nitrophenyl groups in which the linking groups are saturated carbon chain. The molecules chosen for the present study are N[3-(naphthalene-1-yloxy)butyl] -4-nitroaniline (1) and N-[3(naphthalene-1-yloxy)butyl]-2,4-dinitroaniline (2) (Fig.1).

\section{COMPUTATIONAL METHODS}

Gaussian 09 software package was used for DFT calculation and the calculations were performed at four different hybrid density functional theories (DFT) i.e. BPV86, B3LYP, LSDA and $\mathrm{m}-06$ and $6-31++\mathrm{G}(\mathrm{d}, \mathrm{p})$ basis set was used. The gound state structures were optimized and frequency calculations were performed to ensure that the optimized structures are minima in the potential energy surface. HOMO and LUMO for all the molecules are identified. Gauss View 5 software was used for generating the input file and visualization of the results. The calculation were done using S20D300 workstation computer equipped with Intel 7 core processor and 24 GB RAM and Microsoft Windows as the operating system. Electric dipole moment, linear polarizability and first hyperpolarizability tensor components for the studied compounds were calculated by DFT approach which is currently one of the ultimate procedure for obtaining numerically accurate NLO responses.

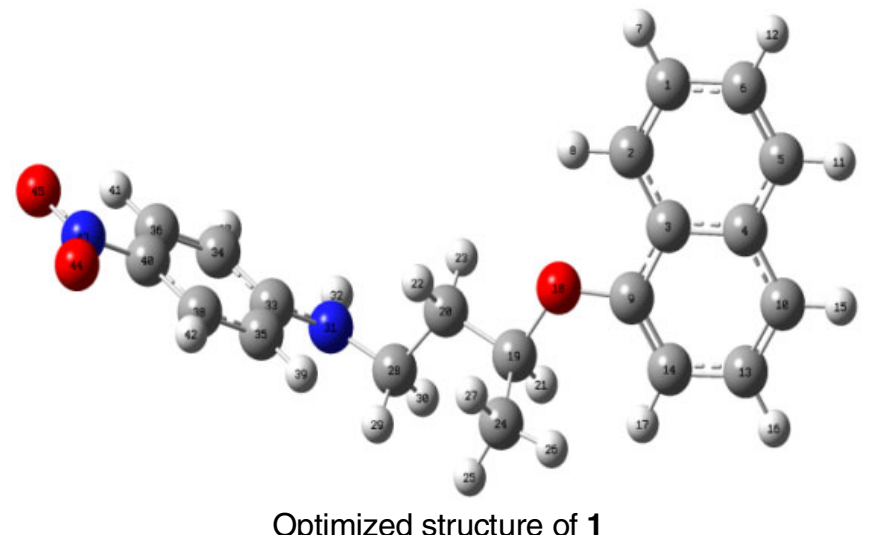

Optimized structure of 1

\section{RESULTS AND DISCUSSION}

Geometry optimization: The optimized molecular geometry represents an isolated molecule under ideal conditions with a stationary point at the potential energy surface. The convergence was confirmed by observing no imaginary vibrational frequencies. The optimized geometries of compounds $\mathbf{1}$ and $\mathbf{2}$ are shown in Fig. 2. Theoretical studies revealed that the compound 1 exists in transoid conformation whereas compound $\mathbf{2}$ exists in cisoid conformation. Analysis of the optimized geometries derived the following observation regarding the structure. The naphthalene part is coplanar with spacer group while the nitrophenyl part of the framework is twisted with respect to the spacer (Fig. 3). This conclusion is supported by the relevant torsional angles listed in Table- 1 .

Frontier molecular orbitals (FMOs): The energies of frontier molecular orbitals viz. HOMO and LUMO, are helpful in investigating the electrical and chemical properties of substrates $[19,20]$. Fig. 3 depicted that the HOMO and LUMO orbitals for compounds $\mathbf{1}$ and $\mathbf{2}$ obtained using B3LYP functionals and 6-31++G(d,p) basis set. The HOMO and LUMOs for two compounds are observed to be identical. HOMO of both compounds

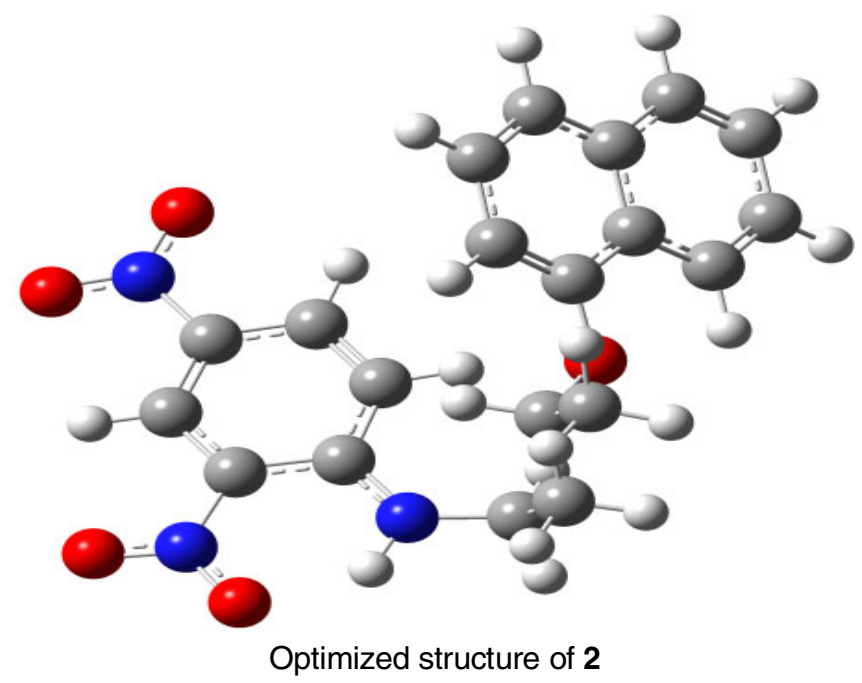

Fig. 2. Optimized geometries of compounds $\mathbf{1}$ and $\mathbf{2}$ 


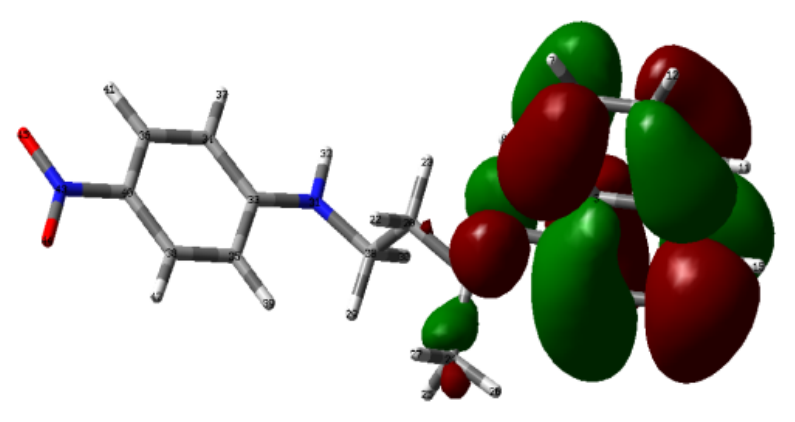

HOMO of 1

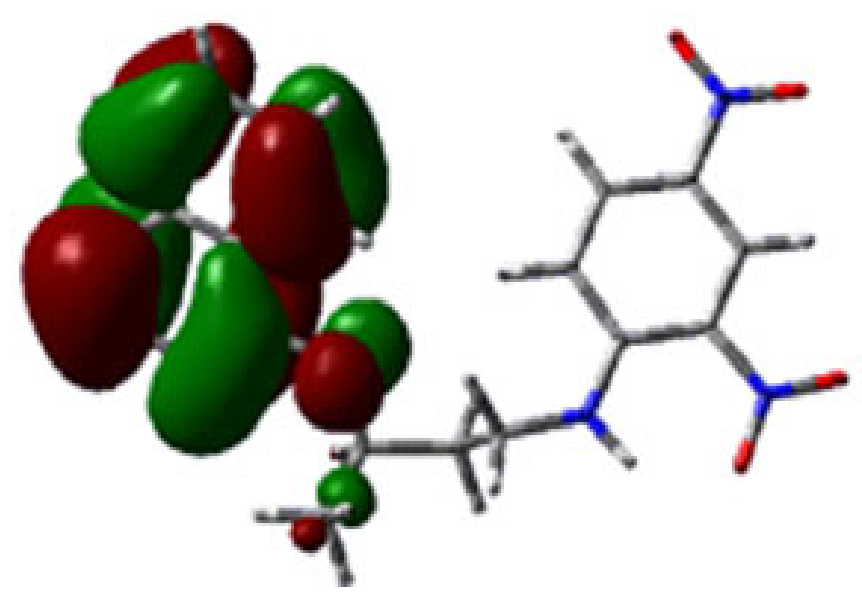

LUMO of 1

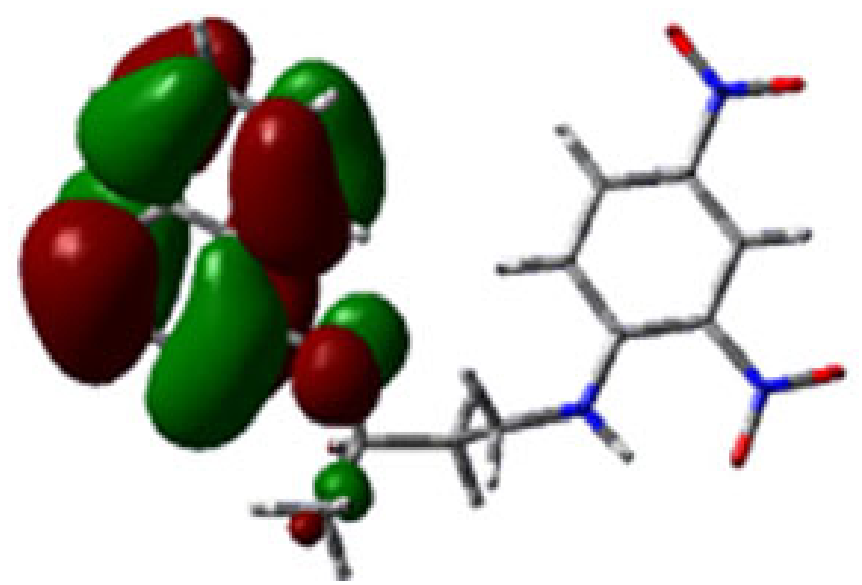

HOMO of 2

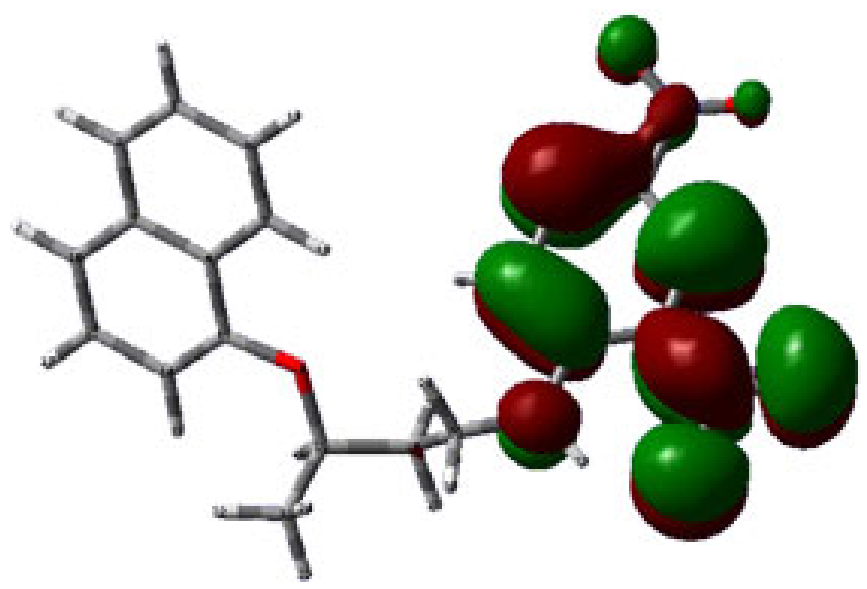

LUMO of 2

Fig. 3. HOMO and LUMO orbitals for compounds $\mathbf{1}$ and $\mathbf{2}$

$\mathbf{1}$ and $\mathbf{2}$ extends over naphthalene donor moieties, whereas LUMO of both compounds $\mathbf{1}$ and $\mathbf{2}$ resides over dinitrophenyl part.

Using the ground state HOMO and LUMO energies, the chemical hardness $(\eta)$, the electronic chemical potential $(\mu)$ and the global electrophilicity index $(\omega)$ have been estimated and the calculated values are listed in Table- 1 .

\begin{tabular}{ccccccc} 
TABLE-1 \\
GROUND STATE HOMO AND LUMO ENERGIES, THE \\
CHEMICAL HARDNESS $(\eta)$, THE ELECTRONIC CHEMICAL \\
POTENTIAL $(\mu)$ AND THE GLOBAL ELECTROPHILICITY \\
INDEX $(\omega)$ OF COMPOUNDS 1 AND 2 \\
\hline \multirow{2}{*}{ Compd. } & $\begin{array}{c}\text { HOMO } \\
\text { (a.u.) }\end{array}$ & $\begin{array}{c}\text { LUMO } \\
\text { (a.u.) }\end{array}$ & $\begin{array}{c}\mathrm{E}_{\mathrm{g}} \\
(\mathrm{eV})\end{array}$ & $\eta$ & $\mu$ & $\omega$ \\
\hline $\mathbf{1}$ & -0.20422 & -0.07029 & 3.64 & 1.82 & 3.73 & 3.83 \\
$\mathbf{2}$ & -0.21101 & -0.09895 & 3.04 & 1.52 & 4.22 & 5.84 \\
\hline
\end{tabular}

Chemical reactivity: The kinetic stability of aforementioned compounds can be monitored by HOMO-LUMO energy gaps [21]. Smaller HOMO-LUMO energy gap leads to lower kinetic stability and higher chemical reactivity. All the present results showed that compound $\mathbf{2}$ is much more reactive than compound $\mathbf{1}$. The chemical reactivity description parameter like chemical hardness $(\eta)$ is useful in studying the stability and reactivity of compounds. It is given in terms of the energies of HOMOs and LUMOs [22]:

$$
\eta=\left(\frac{E_{\mathrm{HOMO}}-\mathrm{E}_{\mathrm{LUMO}}}{2}\right)
$$

This formula indicates that the soft compounds have small chemical hardness, while hard ones have large chemical splitting. The electronic chemical potential $(\mu)$ shows the escaping tendency of electrons in compounds [23] and given by eqn 2 [24,25]:

$$
\mu=-\left(\frac{\mathrm{E}_{\text {HOMO }}+\mathrm{E}_{\mathrm{LUMO}}}{2}\right)
$$

The global electrophilicity index $(\omega)$ estimates the stabilizing energy when a surrounding environment supplies a chemical entity with an additional electronic charge. The index $(\omega)$ relates to the electronic chemical potential $(\mu)$ and the chemical hardness $(\eta)$ through eqn 3 [23]:

$$
\omega=\frac{\mu^{2}}{2 \eta}
$$

The values of global electrophilicity indexes have been calculated and presented in Table-1. It is evident that compound $\mathbf{1}$ is the stronger nucleophile than compound $\mathbf{2}$. It implies that the electron density over compound $\mathbf{1}$ becomes more polarized towards external optical response. Consequently, the degree of delocalization increases which results in higher hyperpolarizability. 
Optical activity: First order polarizability of naphthalene were studied by Hincliffe et al. [26,27] and Howard et al. [28] using different methods and basis sets. In the present study, polarizability of naphthalene is calculated at DFT level using different basis sets. All calculated and experimental values [29] of first order polarizability of naphthalene are enlisted in Table2.

TABLE-2

FIRST ORDER POLARIZABILITY OF NAPHTHALENE AT DIFFERENT LEVELS OF THEORY

\begin{tabular}{lcccc}
\hline \multicolumn{1}{c}{ Model chemistry } & $\alpha_{\mathrm{xx}}$ & $\alpha_{\mathrm{yy}}$ & $\alpha_{\mathrm{zz}}$ & $\begin{array}{c}<\infty> \\
(\mathrm{au})\end{array}$ \\
\hline B3LYP/6-31G(d,p) & 143.66 & 122.81 & 33.31 & 99.92 \\
B3LYP/6-311G(d,p) & 149.41 & 127.73 & 43.09 & 106.74 \\
B3LYP/6-31+G(d,p) & 156.32 & 133.74 & 61.53 & 117.19 \\
B3LYP/6-31++G(d,p) & 156.39 & 133.81 & 62.21 & 117.47 \\
B3LYP/6-311+G(d,p) & 156.30 & 133.64 & 61.86 & 117.26 \\
B3LYP/6-311++G(d,p) & 156.37 & 133.70 & 61.96 & 117.34 \\
B3LYP/6-311++G(2d,p) & 158.35 & 135.49 & 64.25 & 119.37 \\
M-06/6-31+G(d,p) & 144.62 & 123.89 & 33.59 & 100.71 \\
Experimental value & & & & 117.40 \\
\hline
\end{tabular}

It is clear that the mean polarizability value calculated using $6-31++\mathrm{G}(\mathrm{d}, \mathrm{p})$ is in close agreement with experimental value which is available from literature [29]. So DFT method with $6-31++\mathrm{G}(\mathrm{d}, \mathrm{p})$ basis set is selected for NLO calculations. Optical properties such as dipole moment, isotropic linear polarizability $\langle\alpha\rangle$, anisotropic linear polarizability $(\Delta \alpha)$ and first order or static hyperpolarizability $(\beta)$ were calculated using different functionals BPV86, B3LYP, LSDA and M-06. BPV86 uses Perdew's 1986 functional with local correlation replaced by that which was suggested by Vosko et al. [30]; B3LYP is Becke's three parameter exchange functional and the gradient corrected functional of Lee, Yang and Parr [31]; LSDA is the Local Spin Density Approximation and M-06 is Minnesota functional.

The nonlinear optical response of an isolated molecule in an electric field $\mathrm{E}_{\mathrm{i}}$ can be presented as a Taylor series expansion of total dipole moment $\left(\mu_{\mathrm{tot}}\right)$ induced by the field:

$$
\begin{gathered}
\mu_{\lambda}=\left\langle\psi(\mathrm{E})\left|\hat{\mu}_{\lambda}\right| \psi(\mathrm{E})\right\rangle \\
\mu_{\lambda}=\mu_{\lambda}^{0}+\alpha_{\lambda \sigma} \mathrm{E}_{\sigma}+\frac{1}{2 !} \beta_{\lambda \sigma v} \mathrm{E}_{\sigma} \mathrm{E}_{v}+\frac{1}{3 !} \gamma_{\lambda \sigma v \rho} \mathrm{E}_{\sigma} \mathrm{E}_{v} \mathrm{E}_{\rho}+\ldots \ldots . .
\end{gathered}
$$

where, $\alpha$ is the linear polarizability, $\mu_{\mathrm{o}}$ is the permanent dipole moment and $\beta$ is the first hyperpolarizability tensor components. The isotropic (or average) linear polarizability and anisotropy of polarizability [32] is defined as follows:

Isotropic linear polarizability:

$$
\langle\alpha\rangle=\frac{1}{3}\left(\alpha_{x x}+\alpha_{y y}+\alpha_{z z}\right)
$$

Anisotropic linear polarizability:

$$
\Delta \alpha=\frac{1}{2}\left[\left(\alpha_{x x}-\alpha_{y y}\right)^{2}+\left(\alpha_{x x}-\alpha_{z z}\right)^{2}+\left(\alpha_{y y}-\alpha_{z z}\right)^{2}\right]^{1 / 2}
$$

The complete equation for calculating the total static first hyperpolarizability magnitude of Gaussian output [34] is given as follows:

First order hyperpolarizability:

$\beta_{\text {tot }}=\left[\left(\beta_{x x x}+\beta_{x y y}+\beta_{x z}\right)^{2}+\left(\beta_{y y y}+\beta_{y z}+\beta_{y x x}\right)^{2}+\left(\beta_{z z}+\beta_{z x x}+\beta_{z y y}\right)^{2}\right]^{1 / 2}$

The study involves the initial determination of dipole moment,

\begin{tabular}{|c|c|c|c|c|c|}
\hline \multicolumn{6}{|c|}{$\begin{array}{c}\text { TABLE-3 } \\
\text { DIPOLE MOMENT, ISOTROPIC LINEAR POLARIZABILITY, } \\
\text { ANISOTROPIC LINEAR POLARIZABILITY AND STATIC } \\
\text { HYPERPOLARIZIBILITIES OF COMPOUNDS } 1 \text { AND } 2 \\
\text { USING DIFFERENT LEVELS OF THEORY }\end{array}$} \\
\hline Compd. & Parameter & B3LYP & BPV86 & LSDA & M-06 \\
\hline \multirow{4}{*}{1} & $\begin{array}{l}\text { Dipole } \\
\text { moment }\end{array}$ & 6.67 & 6.67 & 6.53 & 6.81 \\
\hline & $<\infty>$ & 232.69 & 242.27 & 256.81 & 242.82 \\
\hline & $\Delta \alpha$ (a.u.) & 268.37 & 289.77 & 230.35 & 292.27 \\
\hline & $\beta_{\text {tot }}$ (a.u.) & 2306.04 & 2474.84 & 2471.07 & 2499.03 \\
\hline \multirow{4}{*}{2} & $\begin{array}{l}\text { Dipole } \\
\text { moment }\end{array}$ & 7.44 & 7.47 & 7.58 & 7.50 \\
\hline & $<\infty$ & 257.07 & 265.89 & 266.34 & 257.22 \\
\hline & $\Delta \alpha$ (a.u.) & 138.60 & 148.78 & 150.15 & 136.62 \\
\hline & $\beta_{\text {tot }}$ (a.u.) & 1467.67 & 1579.02 & 1716.43 & 1125.73 \\
\hline
\end{tabular}
isotropic linear polarizability, anisotropic linear polarizability and static hyperpolarizibilities in the gas phase. The results are compiled in Table-3.

Calculation results showed that both compounds $\mathbf{1}$ and $\mathbf{2}$ have large first hyperpolarizability and consequently have good nonlinear response. Hyperpolarizability of compound $\mathbf{1}$ is greater than compound $\mathbf{2}$. The magnitudes of hyperpolarizibilities depend on chemical reactivity and structural characteristics of the molecule. Degree of electron delocalization between the two rings, naphthalene and nitrophenyl, has an important role in increasing the hyperpolarizibilities. Optical response properties are governed by the increasing of both conjugation length and strength of donor and acceptor groups, planarity of rings with spacer. Degree of electron delocalization between the two rings can be monitored by measuring effective distance between relevant atoms of two rings (C7-C15) and coplanarity can be assessed by the torsional angles between the planes of donor and acceptor subunits (angle between C11-O1-C7-C8 and angle between C17-C15-N1-C14). The aforementioned geometrical parameters (Fig. 1) obtained from the optimized geometries (Fig. 1). The torsional angles and effective distance between two rings are given in Table-4.

It is evident that the effective distance between two rings for mononitro derivatives are larger than dinitro derivatives. As the effective distance increases, it is believed that the extent of delocalization increases. So mononitro compounds are having large $\beta$ values than dinitro compounds.

TABLE-4

COMPARISON OF FIRST HYPERPOLARIZABILITY FOR COMPOUNDS A AND B USING

B3LYP, BPV86, LSDA AND M-06 AND SOME SELECTED GEOMETRICAL PARAMETERS

\begin{tabular}{cccccccc}
\hline Compd. No. & $\beta$ (B3LYP) & $\beta($ BPV86) & $\beta$ (LSDA) & $\beta($ M-06) & C7-C15 $(\AA)$ & C17-C15-N1-C14 $\left(^{\circ}\right)$ & C11-O1-C7-C8 $\left({ }^{\circ}\right)$ \\
\hline $\mathbf{1}$ & 2026.33 & 2223.519 & 2271.065 & 1891.24 & 6.89 & -6.3 & 1.273 \\
$\mathbf{2}$ & 1467.674 & 1579.016 & 1716.427 & 1125.73 & 4.503 & -10.91 & 2.434 \\
\hline
\end{tabular}




\section{Conclusion}

Nonlinear optical (NLO) properties of $\mathrm{N}$-[3-(naphthalene1-yloxy)butyl]-4-nitroaniline (1) and $\mathrm{N}$-[3-(naphthalene-1yloxy)butyl]-2,4-dinitroaniline (2) have been calculated theoretically using four different hybrid density functional theories (DFT) i.e. BPV86, B3LYP, LSDA and M-06 and 6-31++G(d,p) basis set was used. The results showed that these molecular systems have large first static hyperpolarizabilities. In addition, NLO response of these molecular systems decreases dramatically when nitrophenyl is replaced by dinitrophenyl group. This can be attributed to their chemical reactivity and structural characteristics. Firstly, compound $\mathbf{1}$ is more nucleophilic than compound 2 and hence there is a greater delocalization. Secondly, the extent of delocalization has been reaffirmed by the effective distance between two rings, which is greater for compound $\mathbf{1}$. Finally, naphthalene ring of compound $\mathbf{1}$ is more coplanar to spacer group than compound 2, which enhances the delocalization. Since both the compounds chosen for the present study are reported to have a high hyperpolarizability, they can be used for developing NLO materials.

\section{ACKNOWLEDGEMENTS}

The authors acknowledge to UGC, New Delhi, India for the financial support as MRP Grant.

\section{CONFLICT OF INTEREST}

The authors declare that there is no conflict of interests regarding the publication of this article.

\section{REFERENCES}

1. K. Ambujam, K. Rajarajan, S. Selvakumar, J. Madhavan, G. Mohamed and P. Sagayaraj, Opt. Mater., 29, 657 (2007); https://doi.org/10.1016/j.optmat.2005.11.008.

2. K. Meera, R. Muralidharan, R. Dhanasekaran, P. Manyum and P. Ramasamy, J. Cryst. Growth, 263, 510 (2004); https://doi.org/10.1016/j.jcrysgro.2003.11.093.

3. J.L. Bredas, C. Adant, P. Tackx, A. Persoons and B.M. Pierce, Chem. Rev., 94, 243 (1994); https://doi.org/10.1021/cr00025a008.

4. L.R. Dalton, A.W. Harper, R. Ghosn, W.H. Steier, H. Fetterman, M. Ziari, Y. Shi, R.V. Mustacich, A.K.-Y. Jen and K.J. Shea, Chem. Mater., 7, 1060 (1995); https://doi.org/10.1021/cm00054a006.

5. C.J. Raj, S. Dinakaran, S. Krishnan, B.M. Boaz, R. Robert and S.J. Das, Opt. Commun., 281, 2285 (2008); https://doi.org/10.1016/j.optcom.2007.12.019.

6. H.S. Nalwa and S. Miyata, Nonlinear Optical Organic Molecules and Polymers, CRC Press: Boca Raton, p. 611 (1997).

7. P.N. Prasad and D.J. Williams, Introduction to Nonlinear Optical Effects in Molecules and Polymers, Wiley (1991).

8. A. Shanthi, C. Krishnan and P. Selvarajan, J. Cryst. Growth, 393, 7 (2014); https://doi.org/10.1016/j.jcrysgro.2013.12.011.
9. M. Sethuram, G. Bhargavi, M.V. Rajasehakaran, M. Dhandapani and G. Amirthaganesan, Optik, 125, 55 (2014); https://doi.org/10.1016/j.ijleo.2013.06.069.

10. G.A. Babu, R.P. Ramasamy, P. Ramasamy and S. Natarajan, J. Cryst. Growth, 311, 3461 (2009); https://doi.org/10.1016/j.jcrysgro.2009.04.007.

11. I. Osman, Int. J. Mol. Sci., 18, 239 (2017); https://doi.org/10.3390/ijms18020239.

12. R.M. El-Shishtawy, A.M. Asiri, S.G. Aziz and S.A.K. Elroby, J. Mol. Model., 20, 2241 (2014); https://doi.org/10.1007/s00894-014-2241-5.

13. J. Kulhanek and F. Bures, Beilstein J. Org. Chem., 8, 25 (2012); https://doi.org/10.3762/bjoc.8.4.

14. T.D. Kim and K.S. Lee, Macromol. Rapid Commun., 36, 943 (2015); https://doi.org/10.1002/marc.201400749.

15. X. Chen, C. Jia, Z. Wan, J. Zhang and X. Yao, Spectrochim. Acta A Mol. Biomol. Spectrosc., 123, 282 (2014); https://doi.org/10.1016/i.saa.2013.12.072.

16. P. Srinivasan, T. Kanagasekaran and R. Gopalakrishnan, Cryst. Growth Des., 8, 2340 (2008); https://doi.org/10.1021/cg701143n.

17. U. Gubler, S. Concilio, C. Bosshard, I. Biaggio, P. Günter, R.E. Martin, M.J. Edelmann, J.A. Wytko and F. Diederich, Appl. Phys. Lett., 81, 2322 (2002); https://doi.org/10.1063/1.1507834.

18. R.E. Martin, J.A. Wytko, F. Diederich, C. Boudon, J.-P. Gisselbrecht and M. Gross, Helv. Chim. Acta, 82, 1470 (1999); https://doi.org/10.1002/(SICI)1522-2675(19990908)82:9<1470::AIDHLCA $1470>3.0$. CO;2-N.

19. I. Fleming, Frontier Orbitals and Organic Chemical Reactions, Wiley: London, UK, edn 1, pp. 879-880 (1978).

20. E. Kavitha, N. Sandaraganesan and S. Sebastian, Indian J. Pure Appl. Phys., 48, 20 (2010).

21. K.H. Kim, Y.K. Han and J. Jung, Theor. Chem. Acc., 113, 233 (2005); https://doi.org/10.1007/s00214-005-0630-7.

22. R.G. Pearson, J. Chem. Sci., 117, 369 (2005); https://doi.org/10.1007/BF02708340.

23. P.K. Chattaraj and B. Maiti, J. Am. Chem. Soc., 125, 2705 (2003); https://doi.org/10.1021/ja0276063.

24. P.G. Parr and R.G. Pearson, J. Am. Chem. Soc., 105, 7512 (1983); https://doi.org/10.1021/ja00364a005.

25. A.C. Mebi, J. Chem. Sci., 123, 727 (2011); https://doi.org/10.1007/s12039-011-0131-2.

26. A. Hinchliffe and H.J.S. Machado, Electron. J. Theor. Chem., 2, 49 (1997); https://doi.org/10.1002/ejtc.28.

27. A. Hinchliffe, J.J. Perez and H.J.S. Machado, Electron. J. Theor. Chem., 2, 325 (1997); https://doi.org/10.1002/ejtc.63.

28. S.T. Howard, I.A. Fallis and D.J. Willock, Mol. Phys., 97, 913 (1999); https://doi.org/10.1080/00268979909482893.

29. S.H. Vosko, L. Wilk and M. Nusair, Can. J. Phys., 58, 1200 (1980); https://doi.org/10.1139/p80-159.

30. J.P. Perdew, J.A. Chevary, S.H. Vosko, K.A. Jackson, M.R. Pederson, D.J. Singh and C. Fiolhas, Phys. Rev. B, 46, 6671 (1992); https://doi.org/10.1103/PhysRevB.46.6671.

31. H. Soscún, O. Castellano, Y. Bermúdez, C. Toro-Mendoza, A. Marcano and Y. Alvarado, J. Mol. Struct. THEOCHEM, 592, 19 (2002); https://doi.org/10.1016/S0166-1280(02)00222-1.

32. K.S. Thanthiriwatte and K.M.N. de Silva, J. Mol. Struct. THEOCHEM, 617, 169 (2002); https://doi.org/10.1016/S0166-1280(02)00419-0. 